\title{
Screen Printed Conductive Pastes for Biomedical Electronics
}

\author{
Hendrik Berg, Martin Schubert, Sabine Friedrich and Karlheinz Bock \\ Department of Electrical Engineering and Information Technology, \\ Electronics Packaging Laboratory, Technische Universität Dresden, Germany \\ schubert@avt.et.tu-dresden.de
}

\begin{abstract}
This paper describes the evaluation of screen printed materials fabricated with an additive manufacturing process for flexible biomedical applications. Five different conductive polymeric thick film pastes, printed on a polyimide substrate have been investigated. For the intended biocompatible applications, the cytotoxicity of the used materials was tested through adherent cell test. Furthermore, the electrical resistance, the printed structure thickness, the surface energy and roughness have been examined. Additionally, the mechanical resilience of the printed materials was tested through a bending test. During the bending the electrical resistance of printed meander structures could be monitored indicating failures. Two out of five materials were qualified as non-toxic, all of the materials are useable for flexible electronics, as they provide good electrical and mechanical properties.
\end{abstract}

\section{INTRODUCTION}

Traditionally, the standard process for fabrication of printed circuit boards for electronic devices comprises subtractive methods. However, trending in electronics manufacturing are additive methods [1] due to two major reasons. One part is the production of the substrate, which can be done for example by $3 \mathrm{D}$ printing or spin coating. The other part is the manufacturing of conductive paths. For this task inkjet printing, Aerosol-Jet printing, dispensing or screen printing are common methods. One advantage for the use of additive processes is the ecological advantage by using less material. Furthermore, less chemical solvents or caustics are needed. The latter benefit constitutes why additively manufactured electronics could be suitable for biomedical applications, such as implantable pulse oximeter [2] or pressure sensor [3].

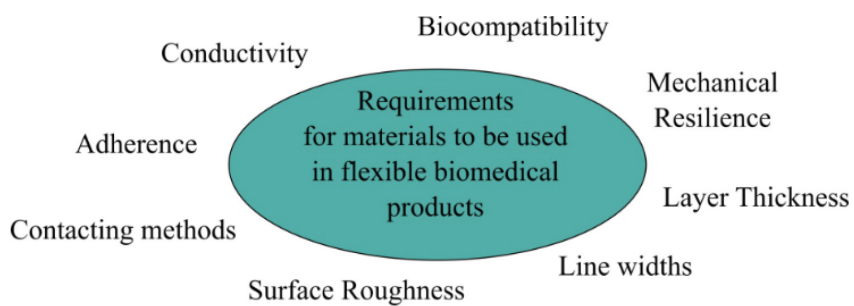

Fig. 1. Requirements for materials in flexible biomedical products
Materials which are going to be used in biomedical products have to meet certain requirements as shown in Fig. 1. For electric circuits, the resistance of the materials used for the buildup of conducting paths is of important matter. Therefore, pastes using silver or copper as conductive particles are suitable as they provide low electrical resistance. However, these materials are known to be toxic and should only be used if hermetic housing is provided [4]. To meet this requirement, rigid hermetical encapsulations of titanium, glass or ceramics are used [4]. However, many applications, such as hook-up electrodes [5], need a flexible assembly. Flexible encapsulations, realized of polymers, do not provide hermetic sealing. Therefore, electric circuits encapsulated by polymeric housings must not be toxic at all if considered for implantable devices. Materials for biocompatible applications therefore have to meet several requirements according to the international standard for biological evaluation of medical devices DIN EN ISO 10993-1. According to this standard, here only the test on cytotoxicity has been carried out. The pastes investigated in this paper shall be used for the buildup of conducting paths. Therefore their electrical resistance has been determined too. Furthermore those pastes are going to be evaluated regarding the properties required in Fig. 1 aiming for an overview of possible material characterization methods. 


\section{Materials ANd Methods}

\subsection{Materials}

Table 1 gives an overview of the used pastes and their conductive parts; the exact material composition is classified by the manufacturers. Due to the thermal stability of the used substrate, which is not stable at temperatures over $360^{\circ} \mathrm{C}$ [6], only pastes with curing temperatures below this temperature were eligible. Except PEDOT all pastes contain a polymeric matrix and a conductive filler, as a PEDOT:PSS ranks amongst intrinsic conductive polymers.

Table 1. Overview of the used materials

\begin{tabular}{ccc}
\hline \hline Abbreviation & $\begin{array}{c}\text { Conductive } \\
\text { Part }\end{array}$ & $\begin{array}{c}\text { Manufacturers } \\
\text { Designation }\end{array}$ \\
\hline C_P_1 & Carbon & DuPont 7102 \\
\hline C_P_2 & Carbon & $\begin{array}{c}\text { Lackwerke } \\
\text { Peters SD 2843 }\end{array}$ \\
\hline Ag_P_1 & Silver & DuPont 5000 \\
\hline CuAg_P & $\begin{array}{c}\text { Copper and } \\
\text { Silver }\end{array}$ & DuPont CB230 \\
\hline PEDOT & PEDOT:PSS & $\begin{array}{c}\text { Heraeus } \\
\text { Clevios }\end{array}$ \\
\hline
\end{tabular}

For the application in biomedical microsystems, these pastes must not be toxic. PEDOT:PSS has already been characterized as not cytotoxic using the L929 mouse fibroblast cell line [7]. Carbon as conductive material within the tested pastes is generally known to be non-toxic. The use of the highly conductive materials copper and silver seems to be contrary to this demand, as these materials are well known to be toxic for cells if present in a higher ionic concentration. Since one objective of this work is to evaluate testing methods for printed conductive structures in common too, Copper and silver pastes have been used as a high conductivity reference compared to carbon or PEDOT. The material DuPont CB230 is solderable and can be used as contact metallization [8], DuPont 5000 is used for low voltage circuitry [9]. All pastes have been printed on a polyimide PI-2611 (HD Microsystems). The polyimide was processed by spin coating on 6-inch Siwafers. After two curing steps $\left(130^{\circ} \mathrm{C}, 2 \mathrm{~min}\right.$ and $350^{\circ} \mathrm{C}$, curing ramp under nitrogen atmosphere for $45 \mathrm{~min})$ a layer thickness of $(9,5 \pm 0,12) \mu \mathrm{m}$ has been reached.

\subsection{Methods}

Several testing methods have been used in this work to characterize printable, conductive materials. Therefore meander shaped structures of three different line widths/line spaces $(150 \mu \mathrm{m} / 150 \mu \mathrm{m}, \quad 250$ $\mu \mathrm{m} / 250 \mu \mathrm{m}$ and $350 \mu \mathrm{m} / 350 \mu \mathrm{m})$ and several pads have been screen printed. The mesh size was $53 \mu \mathrm{m}$ and the thread diameter $24 \mu \mathrm{m}$. The Pads are necessary for characterizing the surface topography and energy as well as the adherence of the printed structures to the substrate. The meander structures are used for mechanical resilience testing and the electrical conductivity measurement of the materials. To determine the layer thickness and surface roughness of the printed materials the confocal microscope $\mu$ surf from Nanofocus AG was applied. The surface energy was determined measuring the contact angle with deionized water.

For materials, which are dedicated for utilization in biomedical products, a major property to characterize is the biocompatibility [10]. Therefore a test of cytotoxicity, which gives some information about the biocompatibility of materials, was carried out using the CellTiter-Blue ${ }^{\circledR}$ (CTB) Cell Viability Assay of Promega GmbH. The cell line HFFF2 was grown on samples of the tested paste, printed on biocompatible glass slides. The samples were put in a well plate and UV-sterilized. Afterwards the samples were incubated for 2 days together with the culture medium. This contains Dulbecco's Modified Eagle's Minimum Essential Medium (DMEM) mixed with Ham's F12 in ratio 1:2 (DMEM/F12) and supplemented with $10 \%$ fetal calf serum (FCS).

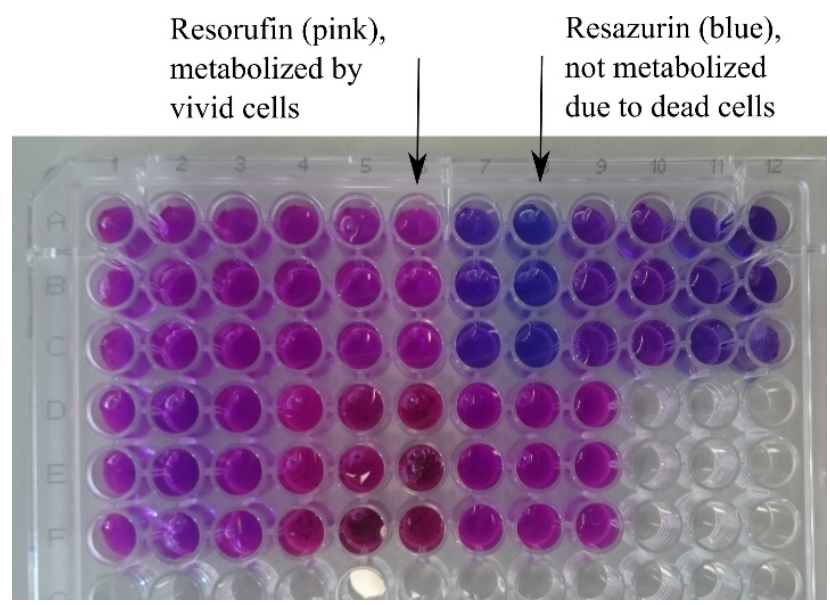

Fig. 4. Change in color of the dye after being incubated with cells for 5 hours 
The CTB assay was applied to the cells and incubated for 5 hours. During this time, Resazurin metabolized into Resorufin but only by vivid cells. This reaction is indicated by a change of color from blue to pink [11]. Depending on the number of living cells on the printed paste the color of the dye varies, as shown in Fig. 4. The cell vitality can be determined through comparison of the absorption spectrum to a positive and negative reference using a photometer.

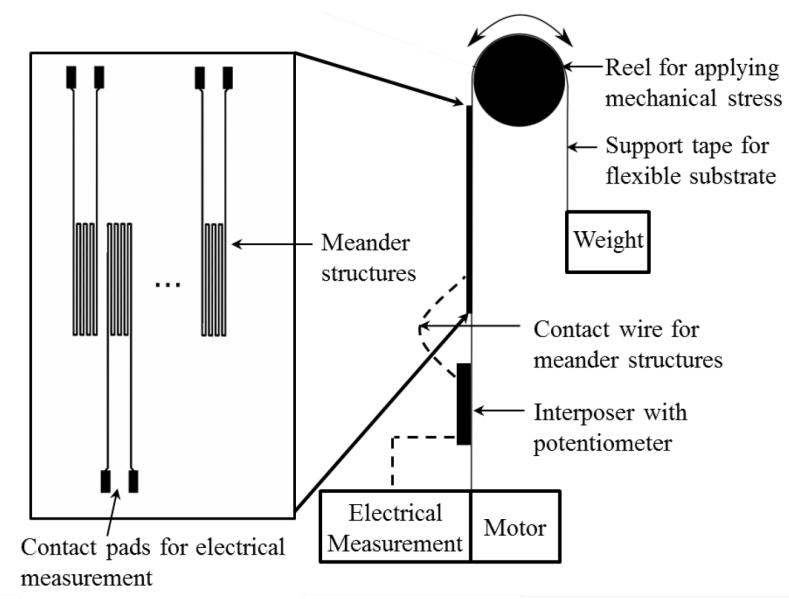

Fig. 5. Test Setup for experiments regarding the mechanical resilience

Furthermore the adherence of printed materials on the substrate has been determined by using the cross-cut or tape test according to the standard DIN EN ISO 2409 , which allows qualitative results by cutting rightangle lattice pattern into the pastes and pull off a tape. The adhesion of the pastes is characterized in a scale between 0 for good adhesion and 5 for no adhesion. The applied tape was Tesapack 4124 PVC.

As flexible microsystems undergo mechanical stress during their lifecycle, the test of mechanical resilience of the printed pastes on the flexible PI substrate was tested. In this work, a bending test has been performed using an existing test setup [12]. The setup (Fig. 5) consists of a reel with certain radius, a support tape, where flexible substrates can be attached for bending, a weight for keeping the tape in tension and a motor, which is controlled via PC and performs the bending movement. Since it's a promising method to detect cracks or failures in conductive tracks through increasing resistance detection $[13,14]$, the described setup was extended for electrical resistance monitoring during the bending experiments. Before the experiments, the resistance of each structure has been measured using a programmable LCR Bridge HM8118 (Rhode \& Schwarz). Bending cycles were performed with a bending radius of $5 \mathrm{~mm}$ or $15 \mathrm{~mm}$ and an angle of 180 degree through changing of the reel. The tests comprised 2700 bending cycles using 5 samples of each material and each line width. Each cycle lasted 30 seconds. The resistance was determined by measuring the electrical voltage of the bended structures while the electric current was adjusted with a potentiometer according to the initial resistance of the meander structure. Therefore a relatively increasing current indicates failure during bending tests performed.

\section{RESUltS}

The results of this work are a series of characterization methods for materials to be used in flexible biomedical electronics. Some of the materials tested during this work are possible to be used in biomedical products in future applications. The results of the cytotoxicity analysis are shown in Fig. 6. For statistics, 9 samples of each material were tested. The red line shows $100 \%$ vitality which has been determined by measuring the absorption spectrum of cells grown on glass slides without paste. The PI substrate used was proved to be non-toxic. Two of the tested pastes, PEDOT:PSS and one of the carbon pastes, attain $100 \%$ or more cell vitality. C_P_2, Ag_P and $\mathrm{CuAg}$ P are below $88 \%$ cell vitality and therefore considered as toxic.

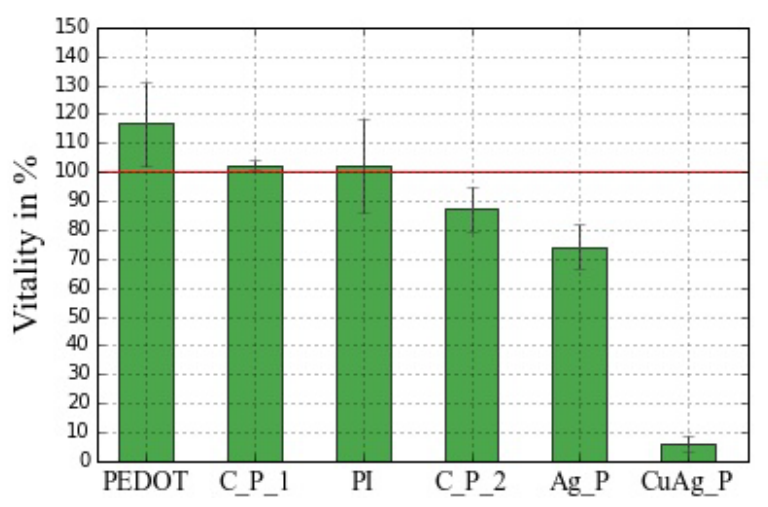

Fig. 6. Cell vitality for measuring cytotoxicity

The results for bending cycles are shown in Fig. 7 and Fig. 8. For each material and line width is the average cycle number of 5 samples shown. Also the minimal cycle number (MIN), which represents the earliest failure and the maximal achieved cycle 


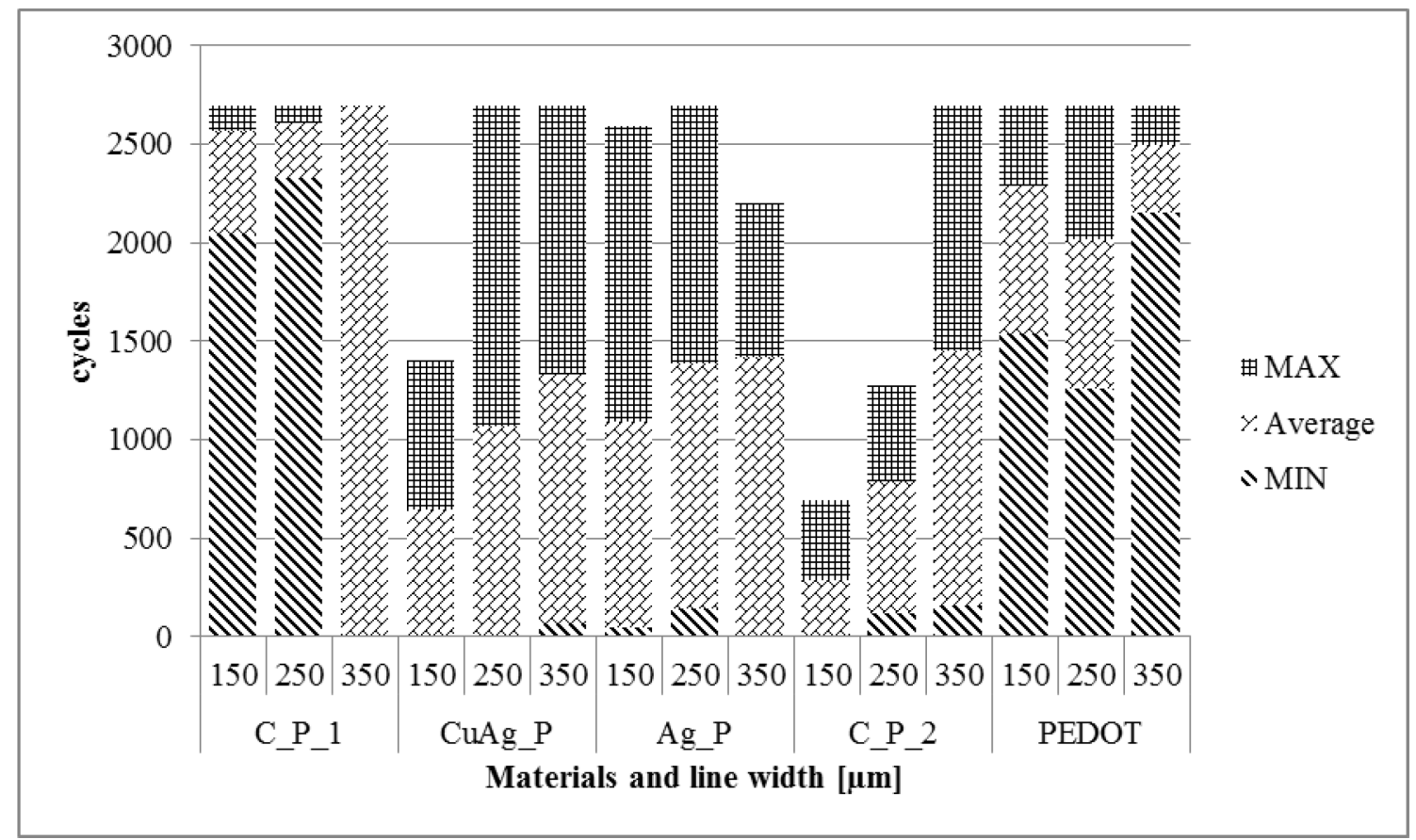

Fig. 7. Bending cycles for each material and line width using the $5 \mathrm{~mm}$ reel

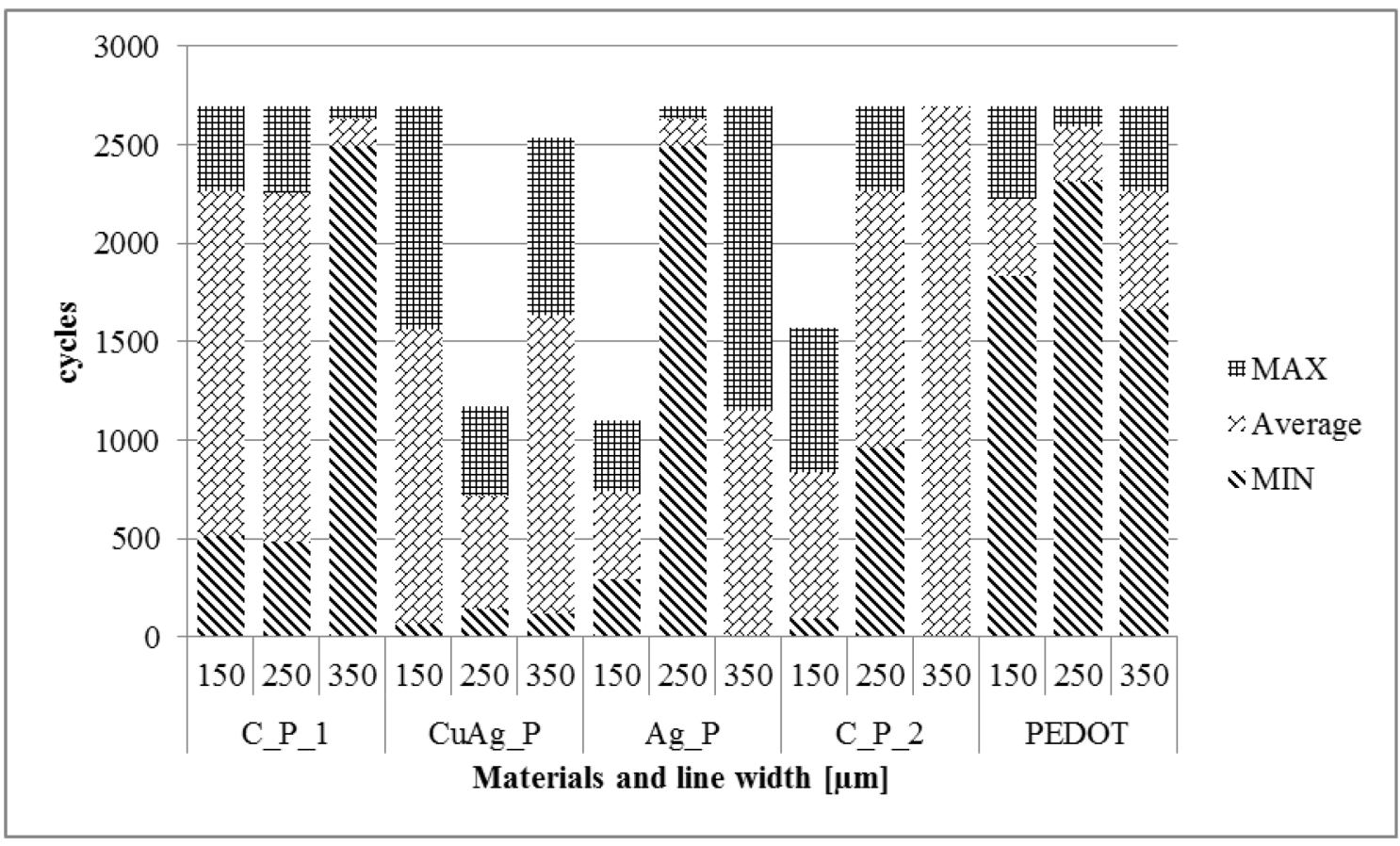

Fig. 8. Bending cycles for each material and line width using the $15 \mathrm{~mm}$ reel

number (MAX) are shown. The highest cycle numbers have been achieved by the non-toxic materials $\mathrm{C}_{-} \mathrm{P} \_1$ and PEDOT. The paste C_P_2 also shows good results for $15 \mathrm{~mm}$ bending radius, not yet for $5 \mathrm{~mm}$. $\mathrm{CuAg} \mathrm{P}$ and $\mathrm{Ag} \mathrm{P}$ seem to be not suitable for bending applications with those radii. Despite the expected higher mechanical stress in smaller radii, no significant difference between the radii can be demonstrated.

Table 2 shows the initial resistivity of each material, which has been measured prior to the test. The lowest resistivity was reached by $\mathrm{Ag} \mathrm{P}$ and $\mathrm{CuAg} \mathrm{P}$. The non-toxic materials and $\mathrm{C} P \mathrm{P} 2$ show a much higher resistivity by factor $10^{2}$ to $10^{3}$. 
Table 2. Electrical resistivity measured prior to bending tests and deviation of designed and printed line widths

\begin{tabular}{|c|c|c|c|}
\hline Material & $\begin{array}{l}\text { Line } \\
\text { width } \\
\text { in } \mu \mathrm{m}\end{array}$ & $\begin{array}{l}\text { Resistivity } \\
\text { in } \\
\Omega \cdot m^{2} / m\end{array}$ & $\begin{array}{c}\text { Deviation of } \\
\text { line width in } \\
\%\end{array}$ \\
\hline \multirow{3}{*}{ C_P_1 } & 150 & 1514,9 & 9,6 \\
\hline & 250 & 1423 & 7,4 \\
\hline & 350 & 1601,5 & 4,8 \\
\hline \multirow{3}{*}{ C_P_2 } & 150 & 4362,6 & 58,9 \\
\hline & 250 & 2920,3 & 22,7 \\
\hline & 350 & 2069 & 27,8 \\
\hline \multirow{3}{*}{ Ag_P } & 150 & 0,8 & 83,9 \\
\hline & 250 & 0,7 & 44,9 \\
\hline & 350 & 0,7 & 32,7 \\
\hline \multirow{3}{*}{$\mathrm{CuAg} \_\mathrm{P}$} & 150 & 5,1 & 35,2 \\
\hline & 250 & 8,3 & 25,3 \\
\hline & 350 & 7,1 & 16,3 \\
\hline \multirow{3}{*}{ PEDOT } & 150 & 10547,5 & 34,1 \\
\hline & 250 & 8662,1 & 15,8 \\
\hline & 350 & 7999,4 & 16,67 \\
\hline
\end{tabular}

Between designed and printed line width a deviation can be dedicated due to surface conditions of the materials. This represents an important aspect, which should already be considered when designing conductive lines for biocompatible electronics. Table 2 shows for each line width the deviation of measured structures related to designed feature sizes. All pastes showed spreading geometry when printed. Smaller line widths show a higher deviation than larger structures. The smallest line width that could be printed during this work has been $164 \mu \mathrm{m}$ using the non-toxic paste $\mathrm{C} P 1$.

Table 3. Mean values of layer thickness, surface roughness and surface energy

\begin{tabular}{lccc}
\hline \hline Material & $\begin{array}{c}\text { Layer } \\
\text { thickness } \\
\text { in } \boldsymbol{\mu \mathbf { m }}\end{array}$ & $\begin{array}{c}\text { Surface } \\
\text { roughness } \\
\mathbf{R}_{\mathbf{a}} \text { in } \boldsymbol{\mu \mathbf { m }}\end{array}$ & $\begin{array}{c}\text { Surface } \\
\mathbf{e n e r g y} \text { in } \\
\mathbf{m N} \mathbf{~ m}\end{array}$ \\
\hline C_P_1 & 11 & 1,31 & 88,37 \\
\hline C_P_2 & 23,9 & 1,26 & 62,82 \\
\hline Ag_P & 6,2 & 0,74 & 60,61 \\
\hline CuAg_P & 28,6 & 1,74 & 54,71 \\
\hline PEDOT & 12,7 & 0,95 & 103,81 \\
\hline
\end{tabular}

Confocal microscopy was used to determine the structure thickness, the surface roughness and energy of the printed materials (Table 3). Those properties are interesting for e.g. multi-layer applications or for adherence of biocompatible encapsulations. The surface roughness and energy contribute to the adherence of additional layer by surface enlargement and better wetting conditions. The highest roughness shows CuAg_P and the lowest Ag_P. The surface energy for PEDOT is the highest, which leads to a good wetting for polar fluids. Even the $\mathrm{Ag}$ _ P with lowest surface energy showed an acceptable wetting behavior for water. The measured layer thickness ranges from 6,2 $\mu \mathrm{m}\left(\mathrm{Ag} \_\mathrm{P}\right)$ until 28,6 $\mu \mathrm{m}(\mathrm{CuAg}$ _P $)$.

The adherence of the printed materials to the substrate has been determined using the cross-cut test. This test has shown, that C_P_1, C_P_2 and CuAg_P provide rather good adhesion with a characteristic adhesion value of 1 or 2 according to DIN EN ISO 2409, in comparison to PEDOT and Ag_P which had characteristic adhesion values of 5 .

\section{Conclusion}

This paper gives an overview to characterization methods for screen printed, conductive structures considered for flexible, biocompatible applications. Key properties investigated were cytotoxicity, resistivity, flexibility, feature size and thickness, surface properties.

Only two of the tested pastes, PEDOT:PSS and one of the carbon pastes can be characterized as nontoxic to the cell line HFFF2. For those materials applications with direct tissue contact to fibroblasts could be considered. The other materials should be encapsulated in hermetic enclosures for application in biocompatible environments. Advantageous other properties such as better conductivity have been reached with the toxic metal based pastes. Yet compared to bulk copper with a resistivity of $0,017 \Omega \cdot \mathrm{mm}^{2} / \mathrm{m}$, all tested materials showed more than factor 40 higher electrical resistivity.

The results of the test for mechanical resilience show, that at minimum one structure of each material and both tested bending radii, achieved the maximum tested number of cycles. The relation between bending radius, layer thickness and feature size has to be further investigated. 
Screen printing therefore proofs as established and well known technology also for biomedical applications, in particular since feature size and printing quality still can be advanced further along with new biocompatible pastes becoming available at present.

\section{ACKNOWLEDGEMENTS}

The authors would like to thank Antje Beyer and Prof. Günter Vollmer from the chair for Molecular Cell Physiology and Endocrinology of TU Dresden for providing the equipment for cytotoxicity tests.

This work was supported by the European Social Fund (ESF) and the Free State of Saxony.

\section{REFERENCES}

[1] P. C. Joshi et al., "Direct Digital Additive Manufacturing Technologies: Path Towards Hybrid Integration", Future of Instrumentation International Workshop (FIIW), 2012

[2] S. Reichelt et al., "Development of an Implantable Pulse Oximeter", IEEE Transactions on Biomedical Engineering, Vol. 55, No. 2, Pt 1, pp. 581-588, 2008

[3] S. Kirsten at al., "Biocompatible packaging for implantable miniaturized pressure sensor device used for stent grafts: Concept and choice of materials", IEEE 16th Electronics Packaging Technology Conference (EPTC), 2014

[4] S. Kirsten, J. Uhlemann, M. Braunschweig, K. J. Wolter, "Packaging of Electronic Devices for LongTerm Implantation", $35^{\text {th }}$ International Spring Seminar on Electronics Technology, 2012

[5] M. Varga, K. Schulz, A. Taschwer, K. Wolter, "Towards wireless neural electrodes: systemintegration for stimulating and recording of nerve signals", Electronics System-Integration Conference, 2014

[6] HD Microsystems, "PI-2600 Series - Low Stress Applications", $\mathrm{http}: / / w w w . d u p o n t . c o m / c o n t e n t / d a m /$ dupont/products-and-services/electronic-and-ectrical- materials/semiconductor-fabrication-and-packagingmaterials/documents/PI-2600_ProcessGuide.pdf, 2009, accessed 02/04/2016

[7] F. Pires, Q. Ferreira, C. A. V. Rodrigues, J. Morgado, F. Castelo Ferreira, "Neural stem cell differentiation by electrical stimulation using a cross-linked PEDOT substrate: Expanding the use of biocompatible conjugated conductive polymers for neural tissue engineering, Biochimica et Biophysica Acta, Vol. 1850, No. 6, pp. 1158 - 1168, 2015

[8] DuPont, "Screen Printed Inks for Printed Circuit Boards", http://www.dupont.com/products-andservices/electronic-electrical-materials/printed-circuitboard-materials/brands/screen-printed-inks-forpcb.html, accessed 02/04/2016

[9] DuPont, "DuPont 5000 Technical data Sheet", http://www.dupont.com/content/dam/dupont/productsand-services/electronic-and-electricalmaterials/documents/prodlib/5000.pdf, accessed $02 / 04 / 2016$

[10] E. Wintermantel, S.-W. Ha, "Medizintechnik", Springer, 2009

[11] Promega Corporation, "CellTiter-Blue $\subset$ Cell Viability Assay Technical Bulletin", https://www.promega.de/resources/protocols/technical -bulletins/101/celltiter-blue-cell-viability-assayprotocol/, accessed 02/04/2016

[12]D. Ernst, T. Zerna, K. J. Wolter, "Influences of organic materials on packaging technologies and their consideration for lifetime evaluation", $34^{\text {th }}$ International Spring Seminar on Electronics Technology (ISSE), 2011

[13]F. Molina-Lopez et al., "Study of bending reliability and electrical properties of platinum lines on flexible polyimide substrates", Microelectronics Reliability, Vol. 54, No. 11, pp. 2542-2549, 2014

[14]B.-J. Kim et al., "Crack nucleation during mechanical fatigue in thin metal films on flexible substrates", Acta Materialia, Vol. 61, No. 9, pp. 3473-3481, 2013 